\title{
Investigation into the mechanism of action of the antimicrobial peptides Os and Os-C derived from a tick defensing
}

\author{
Helena Taute ${ }^{\mathrm{a}}$, Megan J. Bester ${ }^{\mathrm{a}}$, Albert W.H. Neitz ${ }^{\mathrm{b}}$, Anabella R.M. Gaspar ${ }^{\mathrm{b}}$ \\ ${ }^{2}$ Department of Anatomy, Faculty of Health Sciences, University of Pretoria, 0002, South Africa \\ ${ }^{b}$ Department of Biochemistry, Faculty of Natural Sciences, University of Pretoria, 0002, South Africa \\ Corresponding author: Helena Taute, Department of Anatomy, Faculty Health Sciences, University of \\ Pretoria, Private Bag x323, Arcadia, 0007, Tel: +27123192695, Fax: +27123192240, \\ helena.taute@up.ac.za
}

\section{Highlights}

- Os and Os-C caused altered intracellular morphology of E. coli and B. subtilis.

- Membrane effects were observed with fluorescence and TEM studies.

- Os and Os-C were able to enter bacterial cells.

- The peptides may have intracellular targets such as DNA.

- Differences observed between Os and Os-C indicate dissimilar modes of action.

\begin{abstract}
Os and Os-C are two novel antimicrobial peptides, derived from a tick defensin, which have been shown to have a larger range of antimicrobial activity than the parent peptide, OsDef2. The aim of this study was to determine whether the peptides Os and Os-C are mainly membrane acting, or if these peptides have possible additional intracellular targets in Escherichia coli and Bacillus subtilis. Transmission electron microscopy revealed that both peptides adversely affected intracellular structure of both bacteria causing different degrees of granulation of the intracellular contents. At the minimum bactericidal concentrations, permeabilization as determined with the SYTOX green assay seemed not to be the principle mode of killing when compared to melittin. However, fluorescent triple staining indicated that the peptides caused permeabilization of stationary phase bacteria and TEM indicated membrane effects. Studies using fluorescently labeled peptides revealed that the membrane penetrating activity of Os and Os-C was similar to buforin II. Os$C$ was found to associate with the septa of $B$. subtilis. Plasmid binding studies showed that Os and Os-C binds $E$. coli plasmid DNA at a similar charge ratio as melittin. These studies suggest membrane activity for Os and Os-C with possible intracellular targets such as DNA. The differences in permeabilization at lower concentrations and binding to DNA between Os and Os-C, suggest that the two peptides have dissimilar modes of action.
\end{abstract}

Keywords: defensin, tick, C-terminus, membrane permeabilization, mode of action, DNA binding.

\section{Abbreviations:}

AAPH, 2,2'-azobis(2-amidinopropane) dihydrochloride; AMP, antimicrobial peptide; CFU, colony forming unit; CTC, 5-cyano-2,3-ditolyl tetrazolium chloride; DAPI, 4',6diamidino-2-phenylindole; EDTA, ethylenediaminetetraacetic acid; 5-FAM, 5carboxyfluorescein; FITC, fluorescein 5(6)-isothiocyanate; HPF, high pressure 
freeze; LB, Luria-Bertani; $\mathrm{MBC}$, minimum bactericidal concentration; OD, optical density; TAE, tris-acetate-EDTA; TEM, transmission electron microscopy.

\section{Introduction}

The increase in bacterial resistance to antibiotics is a major cause for concern all over the world [37]. The study of antimicrobial peptides (AMPs) strives to produce viable candidates for new antibiotics with novel mechanisms of action. AMPs are active against a wide range of microbes and the development of resistance against these agents is less frequent than against traditional antibiotics [18]. The mode of bacterial killing of an AMP needs to be fully understood if it is to be developed as an anti-infective agent.

The amphipathic structure of AMPs allows these molecules to be soluble in an aqueous environment, and also to penetrate lipid-rich membranes. In bacteria, the primary target of AMPs is the membrane. However, AMPs which do not kill by permeabilization may kill through a wide range of intracellular mechanisms [14]. Some intracellular targets include the stimulation of autolytic enzymes, the inhibition of DNA synthesis, protein synthesis, cell wall and membrane synthesis $[8,15]$. The use of microscopy may reveal important insights into the killing mechanism. Hartmann and colleagues used electron microscopy to observe that the peptides gramicidin $S$ and PGLa caused damage of the bacterial envelope, disruption of osmoregulation and affected bacterial DNA [12]. Mangoni et al. described a fluorescence triple staining method with which permeability and viability of cells caused by peptides, could simultaneously be visually evaluated [20]. By fluorescently labeling buforin II analogs and observing the location with microscopy, Park and colleagues revealed that substitution or insertion of proline caused the peptide to become membrane-acting [24].

Disadvantages of using AMPs as antimicrobial treatments include susceptibility to proteases, limited knowledge on toxicity profiles and unknown systemic effects as well as the high cost of production [38]. Therefore, natural AMPs are often used as templates to develop structural analogs with decreased size and increased activity. New analogs of an AMP have to then be re-evaluated for activity and mode of action.

OsDef2, a defensin identified in the midgut of the soft tick Ornithodoros savignyi, has been shortened to the C-terminal peptide Os. Os was also modified to Os-C by removing the three Cys residues from the sequence (Table 1) [26]. Both Os and its analog, Os-C, were previously found to be strongly bactericidal to both Grampositive and Gram-negative bacteria [26]. Os was found to be more active with a shorter killing time than Os-C, suggesting different modes of killing for the two peptides. Both peptides caused the collapse of bacterial cell structures and indentation of cell membranes [26]. Os and Os-C were found not to be toxic to mammalian cells at the concentrations tested, and were shown to possess antioxidant activity [26]. However, their bacterial killing mechanism is not yet fully understood. 
Table 1

Properties of synthetic peptides [26]

\begin{tabular}{llrc}
\hline Peptide & Sequence & $\begin{array}{c}\text { Length } \\
\text { OsDef2 }\end{array}$ & $\begin{array}{c}\text { Net } \\
\text { charge }\end{array}$ \\
\hline Os & KGIRGYKGGYCKGAFKQTCKCY & 37 & +6 \\
Os-C & KGIRGYKGGYKGAFKQTKY & 22 & +6 \\
Melittin & GIGAVLKVLTTGLPALISWIKRKRQQ & 19 & +6 \\
\hline
\end{tabular}

The aim of this study was to determine whether the peptides Os and Os-C are mainly membrane acting, or if they affect additional intracellular targets.

\section{Materials and Methods}

\subsection{Bacterial strains}

Escherichia coli (ATCC 700928) and Bacillus subtilis (ATCC 13933) were used. Bacteria were grown aerobically in Luria-Bertani (LB) broth at $37^{\circ} \mathrm{C}$. To obtain bacteria in the mid-logarithmic phase, bacteria were cultured overnight, diluted 100 times in LB broth and proliferated until an $\mathrm{OD}_{600}$ of 0.5 was reached.

\subsection{Preparation of synthetic peptides}

The peptides Os, Os-C as well as 5-carboxyfluorescein (5-FAM)-labeled Os, Os-C and buforin II were obtained from GenScript (New Jersey, USA). The purity and molecular mass of the peptides were determined by reverse-phase HPLC and mass spectrometry, respectively. Dithiothreitol (DTT, $10 \mathrm{nmol}$ ) was added to Os prior to lyophilization. The peptide melittin (Mel) (Sigma Aldrich, South Africa) is a known lytic peptide and was used as a positive control for membrane damage. Stock peptide solutions of $1.2 \mathrm{mg} / \mathrm{mL}$ were prepared in sterile deionized double distilled water. Peptide concentrations were determined by measuring the absorbance (Abs) of tyrosine or tryptophan residues at $280 \mathrm{~nm}$ and using the equation below:

$$
c=\frac{M W \times d f \times A b s}{\text { no.of Tyr } / \operatorname{Tr} p \times \text { Extinction coefficient }}
$$

where $\mathrm{c}$ is the peptide concentration in $\mathrm{mg} / \mathrm{mL}, \mathrm{MW}$ is the molecular weight of the peptide, and $\mathrm{df}$ is the dilution factor. The extinction coefficients of tyrosine and tryptophan are 1200 and $5560 \mathrm{AU} / \mathrm{mmole} / \mathrm{mL}$, respectively [17]. The concentration of the fluorescently labeled peptides was determined with the same equation, by measuring the absorbance of 5-FAM labeled peptide at the excitation wavelength (492 nm) and using its extinction coefficient (78 $000 \mathrm{AU} / \mathrm{mmole} / \mathrm{mL})$ [5].

\subsection{Transmission electron microscopy}

The methods for high pressure freezing (HPF) and freeze substitution were based on the methods described by Venter et al. [35]. E coli and B. subtilis cells in the midlogarithmic phase were adjusted to a cell density of $64 \times 10^{6} \mathrm{CFU} / \mathrm{mL}$ and exposed to a final concentration of $2 \mu \mathrm{M} \mathrm{Mel}$, Os and Os-C for 10 minutes at $37^{\circ} \mathrm{C}$. After 
exposure of selected bacteria to the peptides, the suspension was centrifuged to prepare a dense pellet and $1 \mu \mathrm{l}$ of the bacteria pellet was used to fill the cavity of gold plated flat specimen carriers from Leica Microsystems. This was followed by high pressure freezing using the Leica EM Pact (Leica Microsystems $\mathrm{GmbH}$, Wetzlar, Germany). To remove all water from the samples, freeze substitution (FS) was carried out with the Leica EM AFS2 (Leica Microsystems GmbH, Wetzlar, Germany) in $2 \%$ osmium tetroxide, $0.1 \%$ uranyl acetate and $99.88 \%$ acetone. The samples were kept at $-90^{\circ} \mathrm{C}$ for 42 hours, heated to $-60^{\circ} \mathrm{C}$ over a 15 hour period, left at $-60^{\circ} \mathrm{C}$ for 8 hours, heated to $-30^{\circ} \mathrm{C}$ over a 15 hour period, left at $-30^{\circ} \mathrm{C}$ for a further 8 hours after which the samples were allowed to warm up to room temperature.

Samples were infiltrated with, and embedded in Embed 812 (SPI Supplies, Pennsylvania, USA). Ultra-thin sections $(100 \mathrm{~nm})$ were prepared with the Leica Ultramicrotome (Leica Microsystems $\mathrm{GmbH}$, Wetzlar, Germany). These sections were picked up on copper grids and contrasted with $4 \%$ aqueous uranyl acetate and Reynolds' lead citrate and rinsed with water. The contrasted sections were viewed and images taken on the JEM-2100F transmission electron microscope (JEOL, Tokyo, Japan).

\subsection{SYTOX Green assay}

To quantify membrane permeabilization of $E$. coli and $B$. subtilis caused by the peptides the SYTOX green assay, adapted from Roth et al. was used [27]. Bacterial suspensions in the mid-logarithmic phase of growth were diluted to a cell density of $1 \times 10^{6} \mathrm{CFU} / \mathrm{mL}$ and incubated with SYTOX green (Life Technologies, South Africa) at a final concentration of $0.1 \mu \mathrm{M}$ in $10 \mathrm{mM}$ sodium phosphate (NaP) buffer $\mathrm{pH} 7.4$ for 15 minutes at $37^{\circ} \mathrm{C}$ in a shaking incubator. The bacteria and SYTOX green mixture was then exposed to a concentration range of Mel, Os and Os-C $(0.1-10 \mu \mathrm{M})$ for 1 hour at $37^{\circ} \mathrm{C}$ in a shaking incubator in a black, flat-bottom, polystyrene costar 96 -well plate (Corning, New York, USA). The plate was then transferred to the SpectraMax Paradigm microplate reader (Molecular Devices, California, USA) and the fluorescence of each well measured using an excitation of $488 \mathrm{~nm}$ and emission of $530 \mathrm{~nm}$. The data is presented as mean \pm standard error of mean. Multiple comparisons were tested by one-way ANOVA followed by the Tukey's post hoc test (http://statistica.mooo.com/).

\subsection{Localization of peptides}

To determine whether Os and Os-C are able to enter intact $E$. coli and B. subtilis, cells exposed to fluorescently labeled peptide were observed with confocal fluorescence microscopy. Mid-logarithmic phase E. coli and B. subtilis were adjusted to a cell density of $64 \times 10^{6} \mathrm{CFU} / \mathrm{mL}$ and exposed to a final concentration of $7.6 \mu \mathrm{M}$ of 5FAM-Os, 5FAM-Os-C and 5FAM-buforin II for 2 hours at $37^{\circ} \mathrm{C}$ in a shaking incubator. Buforin II is a non-membrane acting AMP that is known to cross the cell membrane and was used as a positive control [23]. The bacterial cells were immobilized onto poly-L-lysine coated cover glass slides which were placed in a 24well Cellstar polystyrene plate (Greiner Bio-One GmbH, Kremsmünster, Austria). The cover glass slides were rinsed with $\mathrm{NaP}$ buffer $\mathrm{pH} 7.4$, mounted on glass slides with antifade mounting medium and viewed with the Zeiss LSM 510 Meta Confocal 
Microscope (Carl Zeiss NTS GmbH, Oberkochen, Germany). For visualization, an excitation wavelength of $490 \mathrm{~nm}$ and emission wavelength of $520 \mathrm{~nm}$ was used.

\subsection{Gel retardation assay}

To investigate the effect of peptides on plasmid DNA (pDNA), $2.5 \mu \mathrm{l}$ of $10 \mu \mathrm{g} / \mathrm{mL}$ pBR322 vector from E. coli (Sigma-Aldrich, South Africa) was exposed to $2.5 \mu \mathrm{l}$ of different concentrations of $\mathrm{Mel}$, Os and Os-C for 1 hour at $37^{\circ} \mathrm{C}$. An equal volume of loading solution ( $40 \%$ sucrose and $0.13 \%$ bromophenol blue) was added and analyzed on a $1 \%$ agarose gel in TAE buffer $(0.8 \mathrm{mM}$ Tris, $0.4 \mathrm{mM}$ glacial acetic acid, $10 \mathrm{mM}$ EDTA, pH 8.0). The gel was post stained with a $3 \mathrm{X}$ staining solution of GelRed (Biotium, California, USA) for 1 hour, and imaged with the UVIdoc HD5 gel documentation system (Uvitech, Cambridge, UK).

\subsection{Triple fluorescent staining}

The method described by Mangoni et al. [20], using 5-cyano-2,3-ditolyl tetrazolium chloride (CTC), 4',6-diamidino-2-phenylindole (DAPI) and fluorescein 5(6)isothiocyanate (FITC) was used. Stationary phase E. coli and B. subtilis cultures were adjusted to a cell density of $64 \times 10^{6} \mathrm{CFU} / \mathrm{mL}$ in $10 \mathrm{mM} \mathrm{NaP}$ buffer, $\mathrm{pH} 7.4$, and exposed to peptide concentrations of $0.77 \mu \mathrm{M}$ and $3.75 \mu \mathrm{M}$ and concentrations $10 \mathrm{x}$ lower $(0.077 \mu \mathrm{M}$ and $0.37 \mu \mathrm{M})$ for 10 minutes at $37^{\circ} \mathrm{C}$ in a shaking incubator. The cells were also exposed to the positive control, Mel, at a concentration of $2.5 \mu \mathrm{M}$ for 10 minutes at $37^{\circ} \mathrm{C}$ in a shaking incubator. The bacterial suspensions were subsequently incubated with $5 \mathrm{mM}$ CTC in $10 \mathrm{mM}$ phosphate buffered saline (PBS) $\mathrm{pH} 7.4$ for 2 hours at $37^{\circ} \mathrm{C}$ in a shaking incubator. To allow adhesion of cells to the surface of poly-L-lysine coated cover glass slides, the CTC-bacteria mixture was added to the wells of a 24-well Cellstar polystyrene plate (Greiner Bio-One $\mathrm{GmbH}$, Kremsmünster, Austria) containing the cover glass slides and incubated at $30^{\circ} \mathrm{C}$ for $90 \mathrm{~min}$. The cover glass slides were rinsed with NaP buffer, then DAPI $(10 \mu \mathrm{g} / \mathrm{mL}$ in PBS) was added and incubated at $30^{\circ} \mathrm{C}$ for $30 \mathrm{~min}$. The cover glass slides were rinsed with $\mathrm{NaP}$ buffer, FITC solution $\left(6 \mu \mathrm{g} / \mathrm{mL}\right.$ in PBS) added and incubated at $30^{\circ} \mathrm{C}$ for $45 \mathrm{~min}$. Thereafter the cover glass slides were rinsed with $\mathrm{NaP}$ buffer again, mounted on slides with antifade mounting medium and viewed with the Zeiss LSM 510 Meta Confocal Microscope (Carl Zeiss NTS GmbH, Oberkochen, Germany). The excitation and emission wavelengths for CTC are $450 \mathrm{~nm}$ and $630 \mathrm{~nm}$, for FITC are $490 \mathrm{~nm}$ and $520 \mathrm{~nm}$ and for DAPI are $359 \mathrm{~nm}$ and $461 \mathrm{~nm}$, respectively. Images of all three dyes were taken separately and overlaid with the Carl Zeiss AIM LSM imaging software into a single image containing the three color signals.

\section{Results}

\subsection{Ultrastructural effects of peptides}

Transmission electron microscopy (TEM) was used to investigate ultrastructural changes caused by the peptides. Control cells exposed only to water, showed a regular cell shape with intact cell membranes and a homogenous cytoplasm (Fig. 1 A and $\mathrm{E}$ ). The cell content of some of the control cells were slightly pulled away from 
the cell membrane, due to sample preparation. Mel caused clear intracellular changes and membrane ruffling (Fig. 1B and F). The cytoplasm of cells exposed to Mel contained electron dense fibers interspersed with electron translucent areas. $E$. coli cells exposed to Os and Os-C generally showed retraction of the intracellular content accompanied by indentation of the cell envelope (Fig. 1C). Os-C exposed cells presented with a granulated or more extremely clumped intracellular content (Fig. 1D). The same effect was observed for Os (not shown). A large number of cells also had membrane ruffling (Fig. 1C and D). B. subtilis cells exposed to Os showed cytoplasmic retraction and the intracellular content appeared granulated (Fig. 1G). Cells exposed to Os-C also presented with granulated or clumped cytoplasm, but very few cells showed cytoplasmic retraction (Fig. 1H).

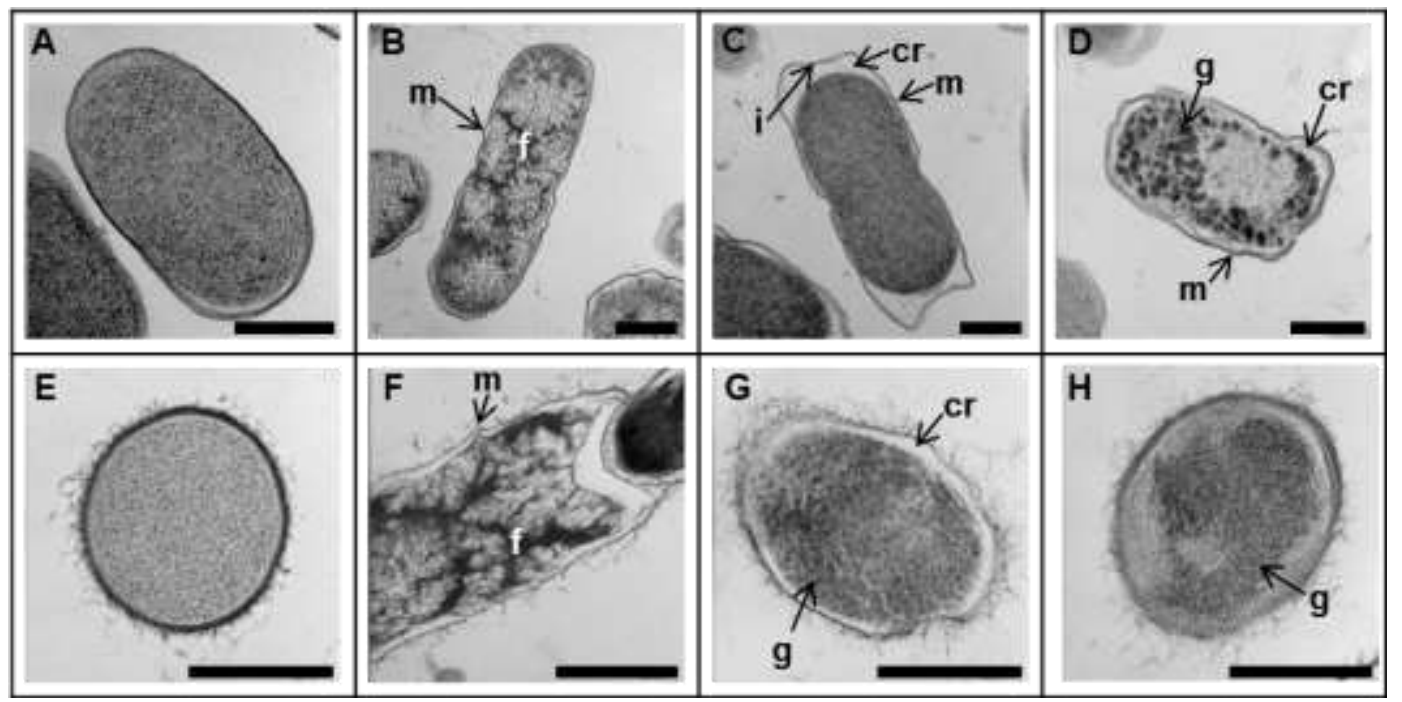

Fig. 1. Ultrastructure of high pressure frozen E. coli $(A-D)$ and $B$. subtilis $(E-H)$ exposed to $2 \mu M$ Mel, Os and Os-C. A, E) Control, B, F) Mel, C, G) Os, D, H) Os-C. cr) cytoplasmic retraction, f) electron-dense fibers, g) intracellular granulation, i) indentation of cell envelope, $\mathrm{m}$ ) membrane ruffling, Scale bars $=500 \mathrm{~nm}$.

\subsection{Membrane permeabilization}

SYTOX green is an unsymmetrical cyanine dye unable to enter intact cell membranes and its fluorescence increases significantly when bound to nucleic acids [27]. If the integrity of the cell membrane is compromised following exposure to the peptides, SYTOX green can enter the bacteria and bind to nucleic acids resulting in an increase in fluorescence emission. Permeabilization of $E$. coli and B. subtilis membranes was measured 1 hour after exposure to the peptides $(0.1-10 \mu \mathrm{M})$. An increase in SYTOX green influx was observed for concentrations below $0.5 \mu \mathrm{M}$ of Os and Os-C, and below $1 \mu \mathrm{M}$ of Mel in both E. coli and B. subtilis (Fig. 2). E. coli cells did not show significant permeabilization at concentrations spanning the minimum bactericidal concentrations (MBCs) of Os and Os-C ( $>0.05)$ compared to the control (bacteria and SYTOX green only). The MBCs were previously determined [26]. For all peptides at higher concentrations, the SYTOX green uptake decreased significantly below that of the control. This decrease was much more pronounced in the 5 and $10 \mu \mathrm{M}$ concentrations of Os, than the equivalent Os-C concentrations. In $B$. subtilis cells, Os caused more permeabilization than Os-C at concentrations of 0.2 and $0.5 \mu \mathrm{M}$. The $0.5 \mu \mathrm{M}$ concentration, which is just below the MBC $(0.77 \mu \mathrm{M})$ of Os, caused significant permeabilization $(p<0.01)$. However, $1 \mu \mathrm{M}$ Os and the 
concentrations spanning the MBC of Os-C $(1.74 \mu \mathrm{M})$ did not cause significant permeabilization. Again, there was a decrease in fluorescence at higher peptide concentrations, where Os showed more of a decrease at 5 and $10 \mu \mathrm{M}$ than Os-C.

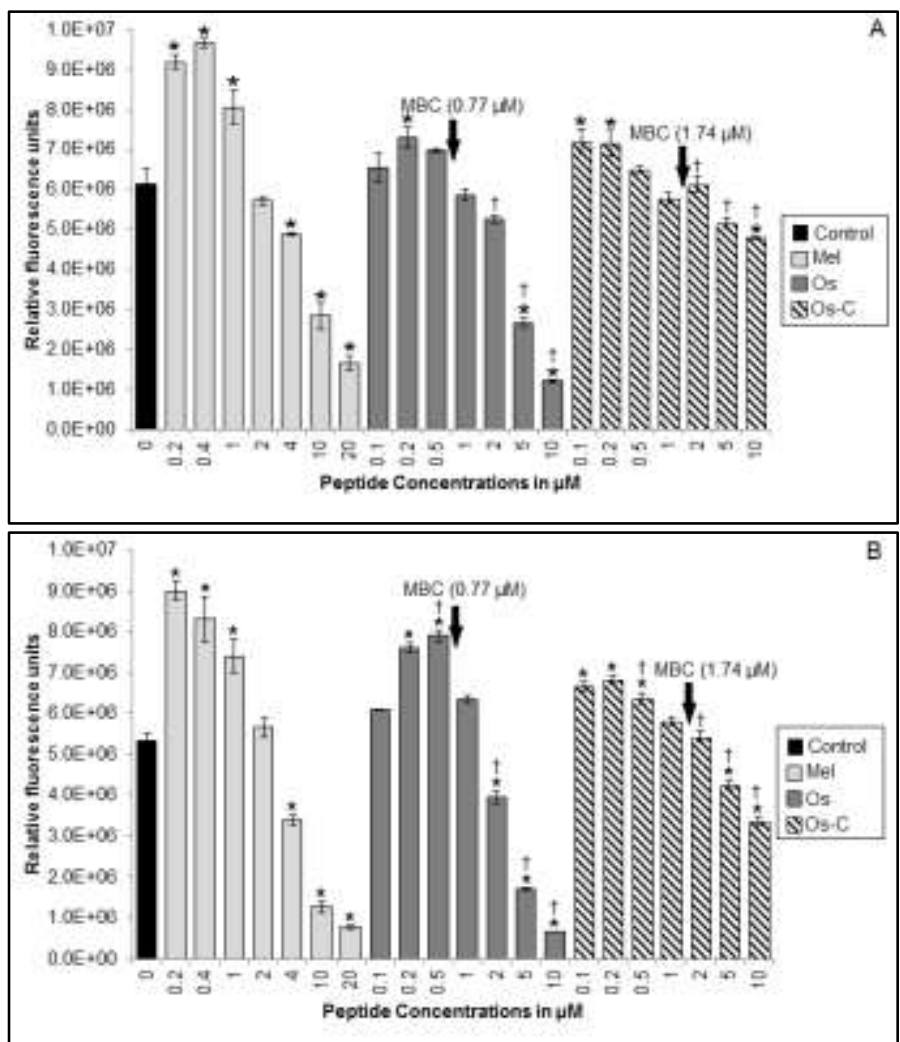

Fig. 2. Membrane permeabilization of A) E. coli and B) B. subtilis exposed to Mel, Os and Os-C for 1 hour. Control is bacteria with SYTOX green only. Permeabilization was measured as an increase in SYTOX green fluorescence (relative fluorescence units) after addition of the peptides. Error bars represent the standard error of the mean of three independent assays. Asterisks indicate significant difference to the control $(p<0.01)$, crosses indicate significant difference between corresponding concentrations of Os and Os-C $(p<0.05)$. Arrows indicate the minimum bactericidal concentrations (MBC) of Os and Os-C.

\subsection{Localization of peptides}

To determine whether the peptides are able to cross membranes, bacteria cells were exposed to fluorescently labeled Os, Os-C and buforin II. The peptides were labeled at the C-terminal with the fluorescent molecule 5-FAM which fluoresces green. Both $E$. coli and B. subtilis were exposed to $7.6 \mu \mathrm{M}$ concentrations of the 5-FAM-labeled Os, Os-C and buforin II for 10 minutes and then counter stained with DAPI. Cells exposed to buforin II showed green fluorescence consistent with a cell-penetrating peptide (Fig. 3A and D) [23, 24]. Cells exposed to labeled Os and Os-C also fluoresced green (Fig. 3B, C, E and F). Some of the septa of B. subtilis cells exposed to Os-C were stained, while in other cells the whole cell stained green. 


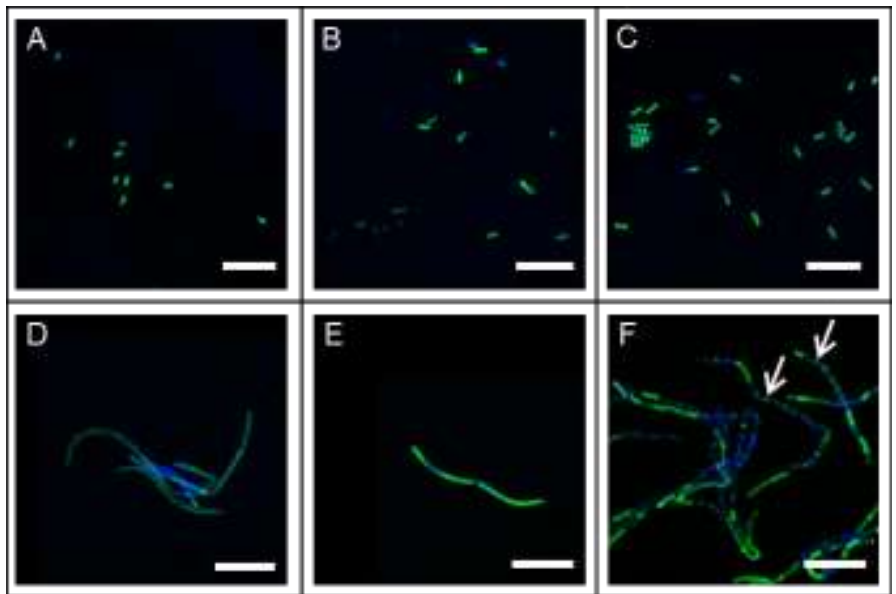

Fig. 3. E. coli and B. subtilis exposed to A, D) 5FAM-buforin II, B, E) 5FAM-Os, and C, F) 5FAM-Os$\mathrm{C}$ (green) and counter stained with DAPI (blue). A, B, C) E. coli and D, E, F) B. subtilis. Arrows indicate stained septa in $B$. subtilis. Scale bars $=10 \mu \mathrm{m}$.

\subsection{Gel retardation assay}

The gel retardation assay is used to determine if peptides bind to plasmid DNA. Ionic interactions between the cationic peptide and plasmid DNA reduce the charge on the plasmid and subsequently the migration is retarded. Additionally peptide binding can cause quenching of plasmid dye fluorescence or may cause the transformation of supercoiled plasmid DNA to the open circular and linear forms as an indication of DNA damage $[9,19,34]$. A single-strand break results in a relaxed circular form of DNA, and a double strand break results in a linear form of DNA [36]. These three forms of DNA travel at different speeds in a gel, and would be seen as bands in different locations. Most supercoiled DNA samples contain some degree of circular DNA depending on the batch [36]. This can be seen in the negative control in lane 1 of Fig. 4. Plasmid in lane 2 and 3 was exposed to $2.5 \mathrm{mM}$ and $5 \mathrm{mM} \mathrm{2,2'-azobis(2-}$ amidinopropane) dihydrochloride (AAPH), respectively, and this resulted in an increase in the amount of circular or linear DNA, indicated as band B [36].

Several studies report the interaction between plasmid DNA and peptide as weight ratios whereas charge ratios, as used in this study, would provide a correct stoichiometric indication of the interaction that occurs. However, in order to compare the present findings to other studies, the weight ratios are also reported. The migration position of the plasmid DNA was unaltered, and remained supercoiled following exposure to Mel at a charge per pmol ratio of 1:0.2 (pDNA:peptide) (lane 4). Exposure to Mel at a charge ratio of 1:0.8 resulted in the formation of an additional band, band $C$, close to the well in lane 5 which indicates bound pDNA. In lane 6 (Mel 1:1.6) and lane 7 (Mel 1:8.3), bands A and B were absent and only band $\mathrm{C}$ was present, and the intensity of the band in lane 7 was reduced. Os and Os-C had no effect on plasmid structure at charge ratios of 1:0.1 and 1:0.5 (lane 8, 9, 12 and 13). However, at charge ratios of $1: 1$ and 1:5, there was a loss of bands $A$ and $B$ and formation of band $C$ (lane 10,14 and 15). Os at a charge ratio of 1:5 showed no staining (lane 11). 


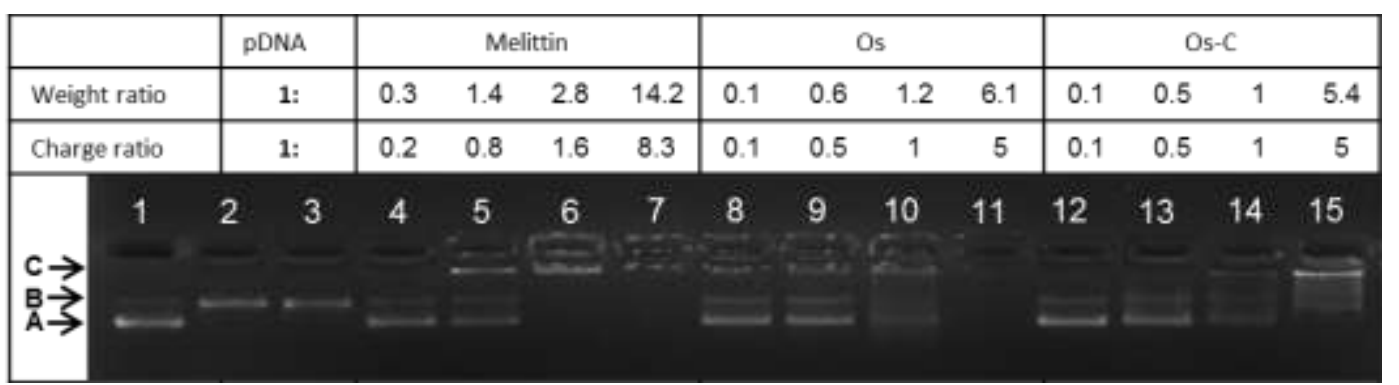

Fig. 4. pBR322 vector from E. coli exposed to AAPH, Mel, Os and Os-C on an agarose gel stained with GelRed. 1) dd $\mathrm{H}_{2} \mathrm{O}$, 2) $2.5 \mathrm{mM} \mathrm{AAPH}$, 3) $5 \mathrm{mM} \mathrm{AAPH}$, 4) $1 \mu \mathrm{M}$ Mel, 5) $5 \mu \mathrm{M} \mathrm{Mel,} \mathrm{6)} 10 \mu \mathrm{M}$ Mel, 7) $50 \mu \mathrm{M} \mathrm{Mel}$, 8) $0.5 \mu \mathrm{M}$ Os, 9) $2.5 \mu \mathrm{M}$ Os, 10) $5 \mu \mathrm{M}$ Os, 11) $25 \mu \mathrm{M}$ Os, 12) $0.5 \mu \mathrm{M}$ Os-C, 13) $2.5 \mu \mathrm{M}$ Os-C, 14) $5 \mu \mathrm{M}$ Os-C, 15) $25 \mu \mathrm{M}$ Os-C. A) Supercoiled pDNA, B) circular / linear pDNA, C) bound pDNA. The weight ratio and charge per pmol ratio (pDNA:peptide) is indicated above lanes $4-15$.

\subsection{Fluorescence triple stain}

In order to visualize membrane permeabilization and the effect on bacterial viability of Os and Os-C, a combination of fluorescent dyes were used. The triple-stain enables the simultaneous viewing of total (stained by DAPI) and viable (stained by CTC) cells, as well as cells with altered membrane permeability (stained by FITC). $E$. coli and $B$. subtilis cells were exposed to $0.77 \mu \mathrm{M}$ Os and $1.74 \mu \mathrm{M}$ Os-C and a concentration 10 times lower for 10 minutes, stained with CTC which fluoresces red in viable cells, DAPI (blue) which stains all DNA and FITC (green) which only stains cells with permeabilized membranes [20]. Control cells of both E. coli and B. subtilis showed no permeabilization, and most of the cells fluoresced red, which indicated viability (Fig. 5A and 6A). Cells exposed to $2.5 \mu \mathrm{M}$ Mel had permeabilized membranes, with no viable cells (Fig. 5B and 6B). Cells exposed to low concentrations of $\mathrm{Os}$ and $\mathrm{Os}-\mathrm{C}$ had permeabilized membranes and viable cells, while at the higher concentrations only a few viable cells were present (Fig. 5C-F and $6 \mathrm{C}-\mathrm{F}$ ). The peptides also seemed to cause a higher degree of cellular clumping than observed in the control cultures.

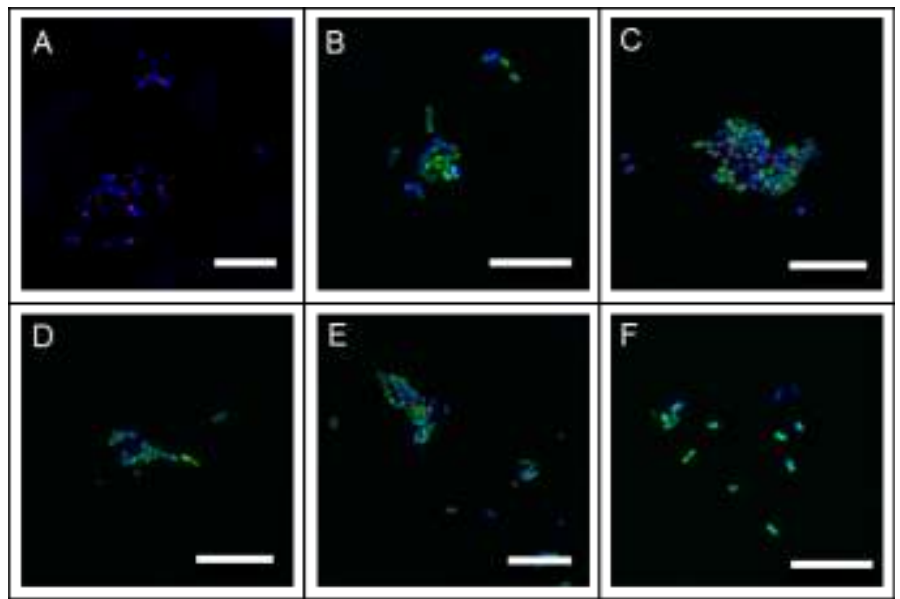

Fig. 5. E. coli in the stationary phase of growth exposed to Mel, Os and Os-C. Cells were stained with DAPI (blue) which stains all DNA, CTC (red) which indicates viability, and FITC (green) which indicates permeabilized membranes. A) Control, B) $2.5 \mu \mathrm{M}$ Mel, C) $0.077 \mu \mathrm{M}$ Os, D) $0.77 \mu \mathrm{M}$ Os, E) $0.174 \mu \mathrm{M}$ Os-C, F) $1.74 \mu \mathrm{M}$ Os-C. Scale bars $=10 \mu \mathrm{M}$. 


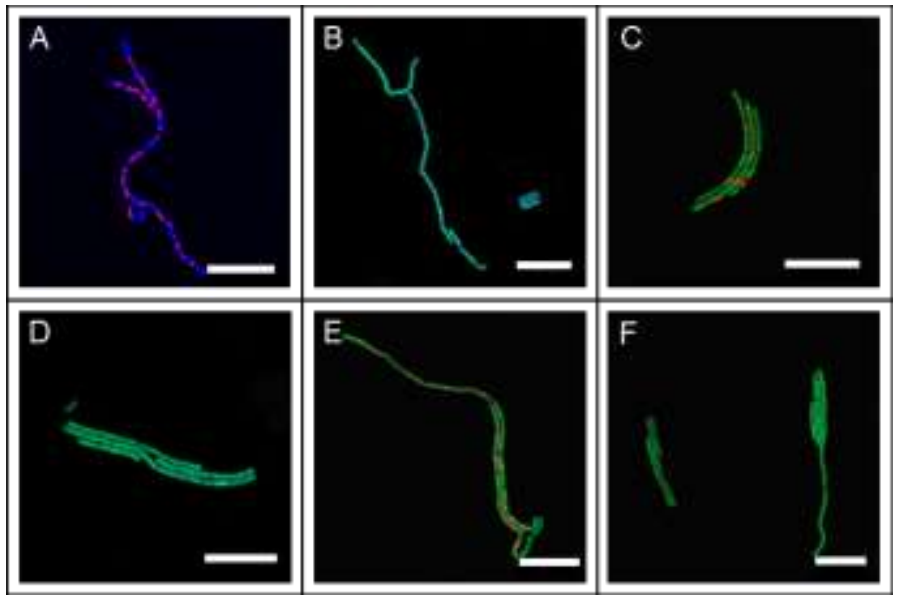

Fig. 6. B. subtilis in the stationary phase of growth exposed to Mel, Os and Os-C. Cells were stained with DAPI (blue) which stains all DNA, CTC (red) which indicates viability, and FITC (green) which indicates permeabilized membranes. A) Control, B) $2.5 \mu \mathrm{M}$ Mel, C) $0.077 \mu \mathrm{M}$ Os, D) $0.77 \mu \mathrm{M}$ Os, E) $0.174 \mu \mathrm{M}$ Os-C, F) $1.74 \mu \mathrm{M}$ Os-C. Scale bars $=10 \mu \mathrm{M}$.

\section{Discussion}

AMPs often serve as templates to develop analogs which are shorter with increased activity. The peptides under investigation were previously found to be active against both Gram-positive and Gram-negative bacteria, whereas the original peptide, OsDef2 was only active against Gram-positive bacteria [26]. The authors also found that Os-C had a slower killing rate than Os, suggesting a differing mode of action [26]. The focus of the present study was to determine if the antimicrobial peptides Os and Os-C are membrane acting, and/or have additional intracellular targets such as the ability to bind DNA. These effects are most probably a function of peptide concentration and exposure time, and the subsequent effects are either immediate, delayed or a combination of both.

TEM studies revealed that bacteria exposed to the peptides had intact membranes, and altered intracellular morphology. The nucleoid was not visible in the HPF controls. However, the formation of electron dense fibers in the Mel exposed bacterial cells is reminiscent of the nucleoid structure observed in chemically fixed bacteria. Therefore, it is likely that the cells exposed to Mel were under enough stress to cause DNA damage. Meincken et al. previously found an increase in the membrane roughness of $E$. coli cells exposed to Mel [22]. The authors attributed this membrane roughness to an increase in the surface area of the membrane, after Mel incorporation into the outer membrane of the bacterial cell [22]. The ruffled appearance observed in the present study may similarly be caused by peptide integration into the membrane. The cells exposed to Os and Os-C did not appear similar to the Mel exposed cells, suggesting a different mode of action. Cells exposed to Os and Os-C showed distinct membrane indentation and cytoplasm which was pulled away from the cell envelope. This correlates with previous scanning electron microscopy results which showed indentations and collapse of the cell structure after exposure to Os and Os-C [26]. The cytoplasmic retraction observed in both $E$. coli and $B$. subtilis is similar to cells undergoing plasmolysis in a hyperosmotic environment $[3,7]$. Pilizota and colleagues found that solutes unable to freely penetrate the outer membrane did not cause plasmolysis, but rather a complete shrinkage of the cell [25]. Only solutes that were outer membrane permeable caused plasmolysis as a result of the difference in osmotic pressure 
between the cytoplasm and periplasmic space [25]. It is therefore possible that Os and Os-C acts on only the outer, but not the inner membrane, resulting in an influx of extracellular fluids into the periplasmic space.

The SYTOX green assay is a commonly used method which measures permeabilization of the inner membranes of bacteria [1]. The peptides caused little permeabilization of cytoplasmic membranes at concentrations spanning the MBC. In $B$. subtilis the $0.2 \mu \mathrm{M}$ and $0.5 \mu \mathrm{M}$ concentrations of Os caused more permeabilization than the equivalent Os-C concentrations, indicating that Os had more membrane activity than Os-C. It was previously observed that cells which were permeabilized by an AMP but not lysed showed a low level of SYTOX green fluorescence as compared to cells which were lysed [27]. It is therefore possible that Os and Os-C permeabilized the cells without causing lysis. This is supported by the triple staining results which indicated membrane permeabilization to FITC. At higher peptide concentrations, the SYTOX green signal decreased to well below that of the control. DNA fragmentation and/or degradation may lead to a decrease in SYTOX green binding which in turn leads to a decrease in fluorescence. Alternatively, the peptides may bind to DNA and compete with SYTOX green for binding sites, which will also lead to a decrease in, or quenching of fluorescence. A similar quenching effect was observed by Tse and colleagues with the fluorescent intercalator displacement assay [33]. In this assay a fluorescent dye (ethidium bromide) binds DNA, which leads to an increase in fluorescence. The addition of a DNA binding compound displaces the bound ethidium bromide, and in turn leads to a decrease in fluorescence [33].

The DNA retardation assay revealed that all peptides caused an inhibition of the migration of pDNA at molar charge ratios (pDNA:peptide) close to or above 1:1. The positively charged peptides most likely formed ionic bonds with the negatively charged DNA. A less negative DNA molecule will result in retarded movement through the agarose gel, which in this case resulted in a third band forming just below the well. In some of the cases, the plasmid DNA stayed partly within the well, or did not migrate indicating completely neutralization of charges on the DNA, e.g. 25 $\mu \mathrm{M} \mathrm{Mel}$ and Os. SYTOX green results also showed a more pronounced decrease in fluorescence for Os than for Os-C. This and the absence of pDNA at $25 \mu \mathrm{M}$ suggest that Os binds more effectively to DNA than Os-C. Buforin II is a non-membrane acting antimicrobial peptide, which has been shown to have a high affinity for DNA and RNA [24]. Buforin II was previously found to bind pDNA at a weight ratio of above 1:0.25 (pDNA:peptide) [23]. Another AMP, indolicidin, has been shown to cause membrane permeabilization without cell lysis, and to have possible intracellular targets through which it kills bacteria [13]. Indolicidin retards the movement of DNA at pDNA:peptide ratios of above 1:0.2 [13]. The peptides Os and Os-C cause pDNA retardation at weight ratios above 1:1.2 and 1:1 (pDNA:peptide), respectively. When comparing the weight ratios, the peptides currently under investigation appear to bind less tightly to pDNA than buforin II or indolicidin. The DNA-binding property can possibly be an explanation for the decrease in SYTOX green fluorescence observed for $E$. coli and $B$ subtilis at high concentrations.

To be able to affect the functioning of an intracellular target, peptides need to cross the cell membrane. Fluorescently labeled peptides which are purely membraneacting will show fluorescence only on the membrane. This phenomenon was 
observed in a study done by Park et al. in 2000, where a fluorescently labeled analog of buforin II was observed on the cell surface, without entering the cell like the original buforin II [24]. In the present study, fluorescence was observed throughout entire cells, indicating the ability of these peptides to cross membranes of both E. coli and B. subtilis. Some septa of $B$. subtilis cells showed green fluorescence, with the adjacent cells unstained, while other cells were completely stained. However, no morphological changes to the septa were observed with TEM. The peptides PR-39 [29], PR-26 [29], indolicidin [31] and microcin [28] were previously found to cause filamentation which may be caused by the inhibition or alteration of membrane proteins required for septum formation [4]. Sochaki and colleagues previously found that fluorescently labeled LL-37 more readily entered $E$. coli cells at the septal regions of dividing cells [30]. However, the research group did not find any evidence for LL-37 preference to septating $B$. subtilis cells [2]. Rapidly dividing $B$. subtilis cultures often lead to the formation of strings of cells as a result of slow septum degradation [10]. As a result of this, the septa of $B$. subtilis cells are visible for a longer time-period than that of $E$. coli. It is possible that Os-C accumulates on $E$. coli septa as well, but it is not observed with the current methodology. The septum peptide accumulation may indicate that Os-C either gains entry into the periplasmic space via the septal region, or that Os-C may have an effect on the function of the cytoplasmic membrane proteins of the septa of B.subtilis. Further studies are necessary to elucidate this septal effect.

During infection, bacteria often encounter less than desirable conditions and periods of limited growth are common [16, 21]. Antimicrobial treatments are often not effective against non-multiplying populations of bacteria, which may lead to clinical relapse of the infection [6]. It is therefore important to determine the activity of a possible antibiotic against non-multiplying or slow multiplying bacteria. For this reason, the effect of $\mathrm{Os}$ and $\mathrm{Os}-\mathrm{C}$ on stationary phase bacteria was investigated. Ten minute exposure to Mel caused membrane permeabilization and associated loss of viability of $E$. coli and $B$. subtilis in the stationary phase, as seen with the fluorescent triple stain. The higher concentrations of $O s$ and Os- $C$ caused membrane permeabilization with only a few viable cells remaining. The results indicated that at low concentrations of Os and Os-C, the peptides cause sufficient membrane permeabilization to allow the penetration of the non-permeable fluorescent dye fluorescein, while the cells remain viable. This indicated that the peptides may act on intracellular targets before bacterial killing occurs. The peptide temporin- $L$ was also previously found to cause membrane permeabilization without cell death at lower concentrations using the triple staining method. The authors concluded that the mechanism of bacterial killing of temporin- $L$ differs at low and high concentrations, and at low concentrations might have intracellular targets [20]. Furthermore, cellular clumping was observed in bacterial cultures exposed to Os and Os-C. Some AMPs were found to cause bacterial clumping or agglutination, which was necessary for bacterial killing [32]. Similarly, Os and Os-C may be forming aggregates on the bacterial cell surface, which leads to cellular agglutination. It is possible that the peptides may aggregate on the bacterial cell surface, where they may interact with the negatively charged surface components and cause partial neutralization. This would cause a disruption of the outer membrane, or cell wall, which would allow the peptides to enter the cell [11, 39]. 


\section{Conclusions}

In this study the mode of bacterial killing of Os and Os-C was investigated. Both Os and Os-C caused granulation of the intracellular contents of $E$. coli and B. subtilis cells, as observed with TEM. The fluorescently labeled peptides were able to enter membranes, and TEM showed that both affect the outer membranes. TEM results also indicated that the peptides may insert into cellular membranes causing membrane permeabilization. The peptides were able to bind to pDNA less tightly than the peptides buforin II and indolicidin. These results point towards membrane acting peptides which may have additional intracellular targets such as DNA. Further studies are necessary to identify other possible intracellular targets, for example, stimulation of autolytic enzymes or inhibition of other proteins essential to the bacterial life cycle [8]. The differences between Os and Os-C, such as the more effective permeabilization of membranes at lower concentrations and binding of Os to DNA, and the accumulation of labeled Os-C on B. subtilis septa, suggest that the two peptides do not act in the same manner.

\section{Acknowledgements}

This work was funded by the Medical Research Council and the National Research Foundation of South Africa. The views and opinions expressed are not those of the MRC and NRF but of the authors. We also thank the Unit for Microscopy and Microanalysis at the University of Pretoria for their technical assistance.

\section{References}

[1] Aragão EA, Chioato L, Ward RJ. Permeabilization of E. coli K12 inner and outer membranes by bothropstoxin-I, A LYS49 phospholipase A2 from Bothrops jararacussu. Toxicon. 2008;51:538-46.

[2] Barns KJ, Weisshaar JC. Real-time attack of LL-37 on single Bacillus subtilis cells. Biochim Biophys Acta Biomembranes. 2013;1828:1511-20.

[3] Bayer M. Response of cell walls of Escherichia coli to a sudden reduction of the environmental osmotic pressure. J Bacteriol. 1967;93:1104-12.

[4] Brogden KA. Antimicrobial peptides: pore formers or metabolic inhibitors in bacteria? Nat Rev Microbiol. 2005;3:238-50.

[5] Buranasompob A. Kinetics of the inactivation of microorganisms by water insoluble polymers with antimicrobial activity. Berlin: Technischen Universität Berlin; 2005.

[6] Coates A, Hu Y, Bax R, Page C. The future challenges facing the development of new antimicrobial drugs. Nat Rev Drug Discov. 2002;1:895-910.

[7] Cota-Robles EH. Electron microscopy of plasmolysis in Escherichia coli. J Bacteriol. 1963;85:499-503.

[8] Cudic M, Otvos JL. Intracellular targets of antibacterial peptides. Curr Drug Targets. 2002;3:101-6.

[9] Ehrenfeld GM, Shipley JB, Heimbrook DC, Sugiyama H, Long EC, Van Boom JH, et al. Copper-dependent cleavage of DNA by bleomycin. Biochem. 1987;26:931-42.

[10] Graumann P. Bacillus: cellular and molecular biology: Horizon Scientific Press; 2012.

[11] Hancock REW, Sahl HG. Antimicrobial and host-defense peptides as new antiinfective therapeutic strategies. Nat Biotechnol. 2006;24:1551-7. 
[12] Hartmann M, Berditsch M, Hawecker J, Ardakani MF, Gerthsen D, Ulrich AS. Damage of the bacterial cell envelope by antimicrobial peptides gramicidin $S$ and PGLa as revealed by transmission and scanning electron microscopy. Antimicrob Agents Chemother. 2010;54:3132-42.

[13] Hsu C-H, Chen C, Jou M-L, Lee AY-L, Lin Y-C, Yu Y-P, et al. Structural and DNA-binding studies on the bovine antimicrobial peptide, indolicidin: evidence for multiple conformations involved in binding to membranes and DNA. Nucleic Acids Res. 2005;33:4053-64.

[14] Izadpanah A, Gallo RL. Antimicrobial peptides. J Am Acad Dermatol. 2005;52:381-90.

[15] Jenssen $H$, Hamill $P$, Hancock REW. Peptide antimicrobial agents. Clin Microbiol Rev. 2006;19:491-511.

[16] Kolter R, Siegele DA, Tormo A. The stationary phase of the bacterial life cycle. Annu Rev Microbiol. 1993;47:855-74.

[17] Lamichhane TN, Abeydeera ND, Duc A-CE, Cunningham PR, Chow CS. Selection of peptides targeting helix 31 of bacterial 16S ribosomal RNA by screening M13 phage-display libraries. Molecules. 2011;16:1211-39.

[18] Lazarev V, Govorun V. Antimicrobial peptides and their use in medicine. Appl Biochem Microbiol. 2010;46:803-14.

[19] Lewis J, Stewart W, Adams D. Role of oxygen radicals in induction of DNA damage by metabolites of benzene. Cancer Res. 1988;48:4762-5.

[20] Mangoni ML, Papo N, Barra D, Simmaco M, Bozzi A, Di Giulio A, et al. Effects of the antimicrobial peptide temporin $L$ on cell morphology, membrane permeability and viability of Escherichia coli. Biochem J. 2004;380:859-65.

[21] Mascio CT, Alder JD, Silverman JA. Bactericidal action of daptomycin against stationary-phase and nondividing Staphylococcus aureus cells. Antimicrob Agents Chemother. 2007;51:4255-60.

[22] Meincken M, Holroyd D, Rautenbach M. Atomic force microscopy study of the effect of antimicrobial peptides on the cell envelope of Escherichia coli. Antimicrob Agents Chemother. 2005;49:4085-92.

[23] Park CB, Kim HS, Kim SC. Mechanism of action of the antimicrobial peptide buforin II: buforin II kills microorganisms by penetrating the cell membrane and inhibiting cellular functions. Biochem Biophys Res Commun 1998;244:253-7.

[24] Park CB, Yi K-S, Matsuzaki K, Kim MS, Kim SC. Structure-activity analysis of buforin II, a histone H2A-derived antimicrobial peptide: the proline hinge is responsible for the cell-penetrating ability of buforin II. Proc Natl Acad Sci. 2000;97:8245-50.

[25] Pilizota T, Shaevitz JW. Plasmolysis and cell shape depend on solute outermembrane permeability during hyperosmotic shock in E. coli. Biophys J. 2013;104:2733-42.

[26] Prinsloo L, Naidoo A, Serem J, Taute H, Sayed Y, Bester M, et al. Structural and functional characterization of peptides derived from the carboxy-terminal region of a defensin from the tick Ornithodoros savignyi. J Pept Sci. 2013;19:325-32.

[27] Roth BL, Poot M, Yue ST, Millard PJ. Bacterial viability and antibiotic susceptibility testing with SYTOX green nucleic acid stain. Appl Environ Microbiol. 1997;63:2421-31.

[28] Salomon R, Farías RN. Microcin 25, a novel antimicrobial peptide produced by Escherichia coli. J Bacteriol. 1992;174:7428-35. 
[29] Shi J, Ross CR, Chengappa M, Sylte MJ, McVey DS, Blecha F. Antibacterial activity of a synthetic peptide (PR-26) derived from PR-39, a proline-arginine-rich neutrophil antimicrobial peptide. Antimicrob Agents Chemother. 1996;40:115-21.

[30] Sochacki KA, Barns KJ, Bucki R, Weisshaar JC. Real-time attack on single Escherichia coli cells by the human antimicrobial peptide LL-37. Proc Natl Acad Sci. 2011;108:E77-E81.

[31] Subbalakshmi C, Sitaram N. Mechanism of antimicrobial action of indolicidin. FEMS Microbiol Lett. 1998;160:91-6.

[32] Torrent M, Pulido D, Nogues MV, Boix E. Exploring new biological functions of amyloids: bacteria cell agglutination mediated by host protein aggregation. PLoS Pathog. 2012;8:1-8.

[33] Tse WC, Boger DL. A fluorescent intercalator displacement assay for establishing DNA binding selectivity and affinity. Acc Chem Res. 2004;37:61-9.

[34] Ueda K, Kobayashi S, Morita J, Komano T. Site-specific DNA damage caused by lipid peroxidation products. Biochim Biophys Acta Gene structure and expression. 1985;824:341-8.

[35] Venter C, Frederick van der Merwe C, Magdalena Oberholzer H, Bester MJ, Taute $\mathrm{H}$. Feasibility of high pressure freezing with freeze substitution after long-term storage in chemical fixatives. Microsc Res Tech. 2013;76:942-6.

[36] Wei Q-Y, Zhou B, Cai Y-J, Yang L, Liu Z-L. Synergistic effect of green tea polyphenols with trolox on free radical-induced oxidative DNA damage. Food Chem. 2006;96:90-5.

[37] Wright GD. Q\&A: Antibiotic resistance: what more do we know and what more can we do? BMC Biol. 2013;11:51.

[38] Yeung ATY, Gellatly SL, Hancock REW. Multifunctional cationic host defence peptides and their clinical applications. Cell Mol Life Sci. 2011;68:2161-76.

[39] Zhao H. Mode of action of antimicrobial peptides: University of Helsinki; 2003. 